\title{
Flightless-1 inhibits ER stress-induced apoptosis in colorectal cancer cells by regulating $\mathrm{Ca}^{2+}$ homeostasis
}

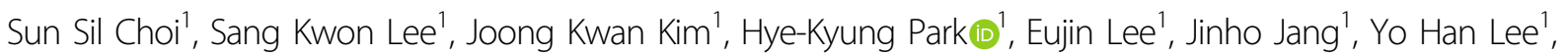 \\ Keon Woo Khim', Ji-Min Hyun'1, Hye-jin Eom', Semin Lee', Byuong Heon Kang ${ }^{1}$, Young Chan Chae', \\ Kyungjae Myung ${ }^{1,2}$, Seung-Jae Myung ${ }^{3}$, Chan Young Park $\mathbb{B}^{1}$ and Jang Hyun Choi ${ }^{1}$
}

\begin{abstract}
The endoplasmic reticulum (ER) stress response is an adaptive mechanism that is activated upon disruption of ER homeostasis and protects the cells against certain harmful environmental stimuli. However, critical and prolonged cell stress triggers cell death. In this study, we demonstrate that Flightless-1 (Flil) regulates ER stress-induced apoptosis in colon cancer cells by modulating $\mathrm{Ca}^{2+}$ homeostasis. Flil was highly expressed in both colon cell lines and colorectal cancer mouse models. In a mouse xenograft model using CT26 mouse colorectal cancer cells, tumor formation was slowed due to elevated levels of apoptosis in Flil-knockdown (Flil-KD) cells. Flil-KD cells treated with ER stress inducers, thapsigargin (TG), and tunicamycin exhibited activation of the unfolded protein response (UPR) and induction of UPRrelated gene expression, which eventually triggered apoptosis. Flil-KD increased the intracellular $\mathrm{Ca}^{2+}$ concentration, and this upregulation was caused by accelerated ER-to-cytosolic efflux of $\mathrm{Ca}^{2+}$. The increase in intracellular $\mathrm{Ca}^{2+}$ concentration was significantly blocked by dantrolene and tetracaine, inhibitors of ryanodine receptors (RyRs). Dantrolene inhibited TG-induced ER stress and decreased the rate of apoptosis in Flil-KD CT26 cells. Finally, we found that knockdown of Flil decreased the levels of sorcin and $\mathrm{ER} \mathrm{Ca}^{2+}$ and that TG-induced ER stress was recovered by overexpression of sorcin in Flil-KD cells. Taken together, these results suggest that Flil regulates sorcin expression, which modulates $\mathrm{Ca}^{2+}$ homeostasis in the ER through RyRs. Our findings reveal a novel mechanism by which Flil influences $\mathrm{Ca}^{2+}$ homeostasis and cell survival during ER stress.
\end{abstract}

\section{Introduction}

Colorectal cancer (CRC) is one of the most common lethal cancers around the world ${ }^{1}$. The disease is characterized by local recurrence, distant metastasis, and extremely rapid progression ${ }^{1}$. Surgical resection, systemic chemotherapy, and radioembolism are the major

\footnotetext{
Correspondence: Sun Sil Choi (ssili77@unist.ac.kr) or Jang

Hyun Choi (janghchoi@unist.ac.kr)

'Department of Biological Sciences, Ulsan National Institute of Science and Technology (UNIST), Ulsan 689-798, Korea

${ }^{2}$ Department of Biological Sciences, Center for Genomic Integrity (CGI), Institute for Basic Science (IBS), Ulsan National Institute of Science and

Technology (UNIST), Ulsan 689-798, Korea

Full list of author information is available at the end of the article

These authors contributed equally: Sun Sil Choi, Sang Kwon Lee
}

strategies for treating CRC, but high mortality and poor prognosis remain serious clinical problems ${ }^{1}$. The main objective of cancer therapy is to destroy all cancer cells while causing minimal damage to normal tissue. Apoptosis, a genetically regulated form of programmed cell death, is an emerging target for anticancer therapy ${ }^{2}$. In general, the activation of apoptosis is an important mechanism for the prevention and treatment of CRC, as well as other cancers ${ }^{2}$. Three different pathways lead to apoptosis: the extrinsic death receptor pathway, the intrinsic mitochondrial pathway, and the endoplasmic reticulum (ER) stress pathway ${ }^{2}$. The mechanisms involved in the intrinsic and ER stress-mediated pathways influence each other. Accordingly, the ER stress pathway 
represents an emerging therapeutic target for cancer treatment ${ }^{3}$.

The ER is a multifaceted organelle involved in initial protein maturation, lipid synthesis, and maintenance of intracellular calcium homeostasis ${ }^{4}$. Disruption of ER homeostasis following glucose deprivation, hypoxia, disruption of $\mathrm{Ca}^{2+}$ homeostasis, or accumulation of misfolded proteins activates the unfolded protein response (UPR) and initiates an intracellular signaling pathway that protects the cell ${ }^{4}$. Under normal conditions, three ER stress sensors, protein kinase-like ER kinase (PERK), activating transcription factor 6 (ATF6), and inositolrequiring kinase-1 (IRE1), are bound to the ER-resident chaperone glucose-regulated protein-78 (GRP78/BiP) ${ }^{5}$. However, under ER stress, these proteins are released from GRP78/BiP and initiate their downstream cascades. IRE1 $\alpha$ initiates the splicing of X-box binding protein-1 (XBP-1) mRNA. Spliced XBP-1 functions as a transcriptional activator of UPR target genes, including GRP78/BiP and calreticulin ${ }^{5}$. PERK is a transmembrane kinase that phosphorylates and inactivates eukaryotic translation initiation factor 2 subunit $\alpha$, leading to reduced protein synthesis ${ }^{5}$. The PERK/EIF1 $\alpha$ pathway also activates ATF4, which upregulates CCAAT/enhancer-binding proteinhomologous protein (CHOP). Concomitantly, during ER stress, cleaved and activated ATF6 $\alpha$ translocates to the nucleus and transactivates the genes encoding various chaperones and ER stress markers, including CHOP and GRP78/BiP itself ${ }^{5}$. Activation of the UPR promotes cell survival by decreasing protein synthesis and increasing protein folding capacity. However, if ER stress persists and homeostasis cannot be restored, the UPR triggers cell death in cells that are beyond repair ${ }^{6}$.

$\mathrm{Ca}^{2+}$ is a ubiquitous intracellular messenger that controls multiple cellular functions, including transcription, exocytosis, apoptosis, and proliferation. $\mathrm{Ca}^{2+}$ is mainly stored in the ER, and its levels are tightly regulated by multiple pumps, channels, and binding proteins in that organelle ${ }^{7} \cdot \mathrm{Ca}^{2+}$ movement across the ER membrane is mediated by $\mathrm{Ca}^{2+}$ release channels, ryanodine receptors (RyRs), ER-resident inositol 1,4,5-trisphosphate receptor $\left(\mathrm{IP}_{3} \mathrm{Rs}\right)$, and the $\mathrm{Ca}^{2+}$ uptake pump sarco-ER $\mathrm{Ca}^{2+}$-ATPase (SERCA $)^{8-10}$. However, despite tight regulation of ER $\mathrm{Ca}^{2+}$, alterations in $\mathrm{ER} \mathrm{Ca}{ }^{2+}$ homeostasis due to dysfunction of these proteins or critical and continuous ER stress provoke ER $\mathrm{Ca}^{2+}$ depletion and an overload of intracellular $\mathrm{Ca}^{2+}$, resulting in excessive $\mathrm{Ca}^{2+}$ accumulation in the mitochondria. This, in turn, causes apoptosis by increasing mitochondrial membrane permeabilization and promoting the release of cytochrome $c^{11}$.

Flightless-1 (FliI), originally identified as a Drosophila melanogaster mutant, is a member of the gelsolin superfamily with an $\mathrm{N}$-terminal leucine-rich repeat domain and a C-terminal gelsolin-like domain ${ }^{12}$. Through its bipartite domain structure, FliI can bind to numerous structural and signaling proteins and thus regulate cell migration, wound healing, and inflammation ${ }^{13-17}$. The main roles of the gelsolin family are $\mathrm{Ca}^{2+}$ - and phosphatidylinositol 4,5bisphosphate-regulated actin binding ${ }^{18}$. However, FliI is more divergent from gelsolin than other family members, and its actin-binding and actin-severing activities are $\mathrm{Ca}^{2+}$ independent ${ }^{19}$. By contrast, FliI interacts in a $\mathrm{Ca}^{2+}$-dependent manner with nonmuscle myosin IIA, which plays an essential role in cell extension by activating transient receptor potential cation channel subfamily $\mathrm{V}$ member $4^{20}$. Furthermore, FliI modulates cell proliferation and survival in cancer cells by interacting with transcription factors such as androgen receptor, estrogen receptor (ER), and carbohydrate responsive elementbinding protein, which regulate tumor progression in prostate cancer and CRC cells ${ }^{21-23}$. Recently, FliI was shown to promote breast cancer progression by impeding selective autophagy through an interaction with $\mathrm{p} 62^{24}$. Here, we report a novel function of FliI: FliI suppresses ER stress-induced UPR signaling and apoptosis in colon cancer by regulating $\mathrm{Ca}^{2+}$ homeostasis through modulation of RyR activity.

\section{Materials and methods}

\section{Cell culture, stable cell line generation}

CT26 (ATCC: CRL-2368) cells were cultured in RPMI 1640 medium supplemented with $10 \%$ fetal bovine serum (GIBCO BRL, Grand Island, NY, USA) and antibiotics (100 U/ml penicillin and $100 \mathrm{ug} / \mathrm{ml}$ streptomycin). Cells were grown at $37^{\circ} \mathrm{C}$ under a humidified $5 \% \mathrm{CO}_{2}$ atmosphere. The sequence used for the lentiviral shRNA expression vector (pLKO.1; Open Biosystems, Huntsville, AL, USA) targeting FliI was 5'-TTCTAGGTTGTT GTTGGCAGC-3'. For lentivirus production, HEK-293T cells (ATCC; Manassas, VA, USA) were transfected with $10 \mu \mathrm{g}$ lentiviral vectors. Following infection with lentivirus, cells were selected with $1 \mu \mathrm{g} / \mathrm{ml}$ puromycin.

\section{Live-cell imaging for intracellular calcium}

shRNA-Ctrl or shRNA-FliI CT26 cells were incubated at $37^{\circ} \mathrm{C}$ for $30 \mathrm{~min}$ in media containing $1 \mu \mathrm{M}$ Fluo-4AM (Invitrogen, Carlsbad, CA, USA). After washing with Hank's buffer, cells were analyzed by flow cytometry or imaged on a fluorescence microscope (Olympus, 20x), with excitation and emission wavelengths of 488 and $505 \mathrm{~nm}$, respectively. For analysis using GCaMP6s, shRNA-Ctrl or shRNA-FliI CT26 cells were seeded on a cover glass and transfected with pDEST-mCherryGCaMP6s using Lipofectamine 2000 (Invitrogen, Carlsbad, CA, USA). After $24 \mathrm{~h}$, intracellular $\mathrm{Ca}^{2+}$ was imaged on an IX83 microscope (Olympus) equipped with an Olympus 40× objective lens (oil, NA 1.30), a fluorescent 
lamp (Olympus), a stage controller (LEP), and a CCD camera (Andor, Concord, MA, USA). Images were processed with MetaMorph software (Molecular Devices, San Jose, CA, USA). For ratiometric $\mathrm{Ca}^{2+}$ imaging, cells were pretreated for $2 \mathrm{~h}$ with dantrolene $(50 \mu \mathrm{M}$; Sigma-Aldrich, St. Louis, MO, USA), 2-APB (50 $\mu$ M; Sigma-Aldrich, St. Louis, MO, USA), tetracaine $(50 \mu \mathrm{M}$; Sigma-Aldrich), DBHQ (2,5-di-tert-butylhydroquinone; $1 \mu \mathrm{M}$; Santa Cruz Biotechnologies, Santa Cruz, CA, USA), and loaded with $1 \mu \mathrm{M}$ Fura-2AM (Molecular Probes) for $30 \mathrm{~min}$. Ratiometric $\mathrm{Ca}^{2+}$ imaging at 340 and $380 \mathrm{~nm}$ was performed at room temperature in calcium-free Tyrode's solution (129 mM NaCl, $5 \mathrm{mM} \mathrm{KCl,} 3 \mathrm{mM} \mathrm{MgCl}, 30 \mathrm{mM}$ glucose, and $25 \mathrm{mM}$ HEPES [pH 7.4]) with or without $5 \mu \mathrm{M}$ ionomycin, $1 \mu \mathrm{M}$ thapsigargin (TG), and $50 \mu \mathrm{M}$ DBHQ on an IX81 microscope equipped with an Olympus 40× objective lens (oil, NA 1.30), a fluorescent arc lamp (Lambda LS), an excitation filter wheel (Sutter, Lambda 10-2), a stage controller (ASI, MS-2000), and a CCD camera (Hamamatsu, C10600). Images were processed using MetaMorph software (Molecular Devices) and analyzed using Igor Pro software (WaveMetrics, Portland, OR, USA).

\section{Analysis of cell viability and apoptosis induction}

shRNA-Ctrl or shRNA-FliI CT26 cells $\left(1 \times 10^{4}\right.$ cells/ well) were cultured in 96-well plates overnight and then treated with TG for $48 \mathrm{~h}$. To determine cell viability, cells were exposed to 3-(4,5-dimethyl-thiazol-2-yl)2,5 diphenyltetrazolium bromide (MTT; Sigma-Aldrich), and crystallized formazan was quantified by measuring absorbance at $595 \mathrm{~nm}$ on an Infinity M200 microplate reader (Tecan, Männedorf, Switzerland). Absorbance data were normalized against the vehicle control and expressed as percent viability. Alternatively, after treatment with TG, shRNA-Ctrl or shRNA-FliI CT26 cells were stained with annexin V-FITC (Invitrogen) and propidium iodide (PI; Invitrogen) and quantified on a FACSCalibur system (BD Biosciences, San Jose, CA, USA). Data were processed using FlowJo software (BD Biosciences). For colony formation assays, shRNA-Ctrl or shRNA-FliI CT26 cells $\left(1 \times 10^{3}\right.$ cells/well) were treated with TG for $48 \mathrm{~h}$, after which the medium was replaced with drug-free medium, and the cells were incubated for 14 days. The colonies were washed with PBS, fixed with methanol, and stained with crystal violet.

\section{Transient transfection of plasmid DNA}

Mouse sorcin expression plasmids were purchased from Sino Biological (BDA, Beijing, China). Plasmid DNA was transiently transfected into FliI-KD CT26 cell lines using jetOptimus (Polyplus-transfection, Illkirch-Graffenstaden, France). Cells were treated with TG at the indicated concentration to induce ER stress.

\section{$\mathrm{ER} \mathrm{Ca}{ }^{2+}$ measurement}

For ER calcium measurements, cells were transiently transfected with a plasmid encoding the D1ER Cameleon. pcDNA-D1ER was a gift from Amy Palmer \& Roger Tsien (Addgene plasmid \#36325) ${ }^{25}$. ZEN (Zeiss, Oberkochen, Germany) was used to calculate fluorescence intensities from images taken under three conditions: IDD, donor fluorescence intensity excited by an excitation laser $(458 \mathrm{~nm})$; IDA, acceptor intensity under a donor excitation laser; and IAA, acceptor intensity excited by an acceptor excitation laser $(514 \mathrm{~nm})$. The FRET efficiency is defined as follows: ${ }^{26}$ FRET efficiency $=\left(I_{\mathrm{DA}}-\beta \times I_{\mathrm{DD}}-\right.$ $\left.\gamma \times I_{\mathrm{AA}}\right) / I_{\mathrm{AA}}$, where $\beta$ is a bleeding-through coefficient from donor to acceptor channels and $\gamma$ is the ratio of fluorescence intensity of acceptor molecules directly excited by a donor excitation laser to fluorescence intensity of acceptor molecules excited by an acceptor excitation laser.

\section{Immunoblotting}

Samples were lysed in RIPA lysis buffer $(50 \mathrm{mM}$ Tris, $\mathrm{pH}$ 8.0, $150 \mathrm{mM} \mathrm{NaCl}, 1 \% \mathrm{NP}-40,0.1 \%$ SDS, and $0.25 \%$ $\mathrm{N}$-deoxycholate) containing protease and phosphatase inhibitors (Sigma-Aldrich). Equal amounts of protein were separated by SDS-PAGE and transferred onto nitrocellulose membranes (GE Healthcare, MA, USA). Membranes were blocked in $5 \%$ bovine serum albumin blocking buffer and incubated at $4{ }^{\circ} \mathrm{C}$ overnight with specific primary antibodies against phospho-PERK, phospho-IRE $\alpha$, GRP78/BiP, CHOP (Cell Signaling Technology, Danvers, MA, USA), sorcin, and FliI (Abcam, Cambridge, MA, USA). Signals were detected using an ECL detection kit (GE Healthcare, Chicago, IL, USA) and subsequently incubated with horseradish peroxidaseconjugated secondary antibodies (Thermo Fisher Scientific, Waltham, MA, USA).

\section{Gene expression analysis}

Total RNA was isolated from cells or tissues using TRIzol reagent (Invitrogen). RNA was reverse transcribed using the ABI Reverse Transcription kit. Quantitative PCR was performed with SYBR green fluorescent dye on an ABI 9300 PCR instrument. Relative mRNA expression was determined by the $\Delta \Delta \mathrm{Ct}$ method and normalized against TBP mRNA expression.

\section{Colon cancer models}

Colon tissues from $\mathrm{APC}^{\mathrm{min}+}$ - and colitis-induced mouse models of CRC were prepared as previously described ${ }^{27,28}$. Briefly, for colitis-induced CRC, 7- to 8-week-old male mice were intraperitoneally injected with azoxymethane (AOM, $10 \mathrm{mg} / \mathrm{kg}$ body weight) and maintained on a regular diet and water for 7 days. Mice were then subjected to five cycles of dextran sodium sulfate (DSS) treatment, in 
which each cycle consisted of $1.5 \%$ DSS for 7 days followed by a 7-day recovery period with regular water.

\section{Xenograft tumor models}

All experiments involving animals were approved by UNIST (IACUC-12-003-A). shRNA-Ctrl or shRNA-FliI CT26 cells $\left(1 \times 10^{6}\right)$ suspended in sterile PBS $(200 \mu \mathrm{l})$ were injected subcutaneously into the left flanks of 6week-old BALB/c nu/nu male mice (SLC Inc., Hamamatsu, Shizuoka, Japan). Tumor sizes were measured using calipers every 2 days for 17 days. Tumor volume was calculated using the following formula: $V=1 / 2 \times$ $(\text { width })^{2} \times$ length. At the end of the experiment, the animals were euthanized, and tumors were collected for western blotting.

\section{Statistical analysis}

Data are presented as the mean \pm standard errors of the mean as indicated in the figure legends. Comparisons between two groups were made by unpaired two-tailed Student's $t$ tests. $p$ values $<0.05$ were considered statistically significant. Microsoft Excel was used for statistical calculations.

\section{Results}

\section{Flil is upregulated in CRCs}

To investigate the clinical relevance of FliI in CRC, we first examined FliI expression in both CRC cell lines and cancer tissue in the $A P C^{\mathrm{min}+}$ - and colitis-induced mouse model of CRC. FliI expression in CRC cell lines and cancer tissues was significantly higher than that in the corresponding normal cell line and nontumor tissues (Fig. $1 \mathrm{a}, \mathrm{b})$. To directly assess the cell-autonomous function of FliI in mouse CRC, CT26 cells stably transduced with either shRNA-Ctrl or shRNA-FliI lentiviral shRNAs were implanted subcutaneously into immunodeficient mice to form xenograft tumors. In accordance with the results shown in Fig. 1b, knockdown (KD) of FliI significantly repressed xenograft tumor growth (Fig. 1c). Moreover, FliI-KD tumors had elevated levels of cleaved PARP-1 and caspase-3 (Fig. 1d), suggesting that the reduced level of FliI promoted apoptosis in CT26 cells.

\section{Flil inhibits ER stress-induced apoptosis in CT26 cells}

The microenvironment within a solid tumor differs from that of normal tissues; the former is characterized by glucose deprivation, low $\mathrm{pH}$, hypoxia, and an imbalance between production and removal of reactive oxidative stress $^{29}$. All of these environmental factors contribute to ER stress; therefore, cancer cells must find effective ways to adapt and prevent ER stress-induced apoptosis ${ }^{3}$. To elucidate the molecular mechanisms by which FliI-KD promotes apoptosis in the xenograft mouse model, we first determined the effect of FliI on cell survival after treatment with the ER stress-inducing drug TG. Cell viability was assessed by MTT and cell counting assays, which revealed that depletion of FliI decreased cell survival following TG treatment (Fig. 2a, b). Colony forming assays confirmed that Flil was required for cell survival following TG treatment (Fig. 2c). Furthermore, FACS analysis using annexin V-PI labeling revealed that depletion of FliI increased ER stress-induced apoptosis (Fig. $2 \mathrm{~d})$. Consistent with the FACS analysis, depletion of FliI increased the levels of cleaved PARP-1 and caspase-3 following TG treatment (Fig. 2e). Another ER stressinducing drug, tunicamycin (TM), significantly increased apoptosis in FliI-KD cells (Supplementary Fig. 1). Together, these data indicate that FliI inhibits ER stressinduced apoptosis.

\section{Knockdown of Flil sensitizes cells to ER stress}

Disturbances in normal ER functions lead to accumulation and aggregation of unfolded proteins, which initiates an adaptive response, the UPR, to restore normal ER function ${ }^{6}$. Failure to activate the adaptive response results in apoptosis $^{30}$. To obtain insights into the molecular mechanism of action of FliI in ER stress-induced apoptosis, we first evaluated the effect of FliI on the ER stress response. As shown in Fig. 3a, within $3 \mathrm{~h}$ of TG treatment, phosphorylation of PERK and IRE $\alpha$ was triggered more strongly in FliI-KD cells than Ctrl cells. In addition to UPR sensor protein activation, GRP78/BiP and $\mathrm{CHOP}$ levels were also elevated in FliI-KD cells. Similar results were observed after treatment of FliI-KD cells with TM (Supplementary Fig. 2a). Furthermore, the expression of UPR genes, including GRP78/BiP, ATF4, ATF6, and CHOP, was significantly elevated in FliI-KD cells after treatment with TG or TM (Fig. 3b and Supplementary Fig. 2b). These results indicate that KD of FliI made CT26 cells more sensitive to ER stress.

\section{Knockdown of Flil promotes ER stress-induced apoptosis by disrupting intracellular $\mathrm{Ca}^{2+}$ homeostasis}

$\mathrm{Ca}^{2+}$ is a major player in the regulation of apoptosis, both at the early and late stages ${ }^{31}$. Disruption of intracellular $\mathrm{Ca}^{2+}$ homeostasis can induce ER stress-mediated apoptosis in response to various pathological conditions $^{32-34}$. Therefore, we next investigated whether ER stress-induced apoptosis sensitized by KD of FliI influence the intracellular $\mathrm{Ca}^{2+}$ concentration. Using Fluo-4AM, a calcium-sensing dye, we observed that depletion of FliI triggered upregulation of intracellular $\mathrm{Ca}^{2+}$ (Fig. 4a, b). Similarly, using GCaMP6s, the $\mathrm{Ca}^{2+}$ indicator, we found that intracellular $\mathrm{Ca}^{2+}$ was significantly increased in FliIKD cells compared with Ctrl cells (Fig. 4c). Furthermore, 1,2-bis(o-aminophenoxy)ethane- $\mathrm{N}, \mathrm{N},-\mathrm{N}^{\prime}, \mathrm{N}^{\prime}$-tetraacetic acid-acetoxymethyl ester (BAPTA-AM), a highly selective $\mathrm{Ca}^{2+}$ chelator, abrogated TG-induced apoptosis in FliI- 


\section{a}

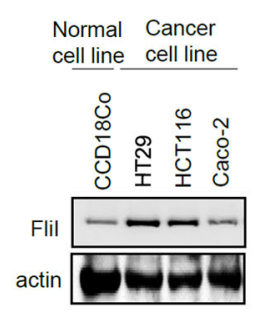

C Ctr ShRNA

Flil shRNA
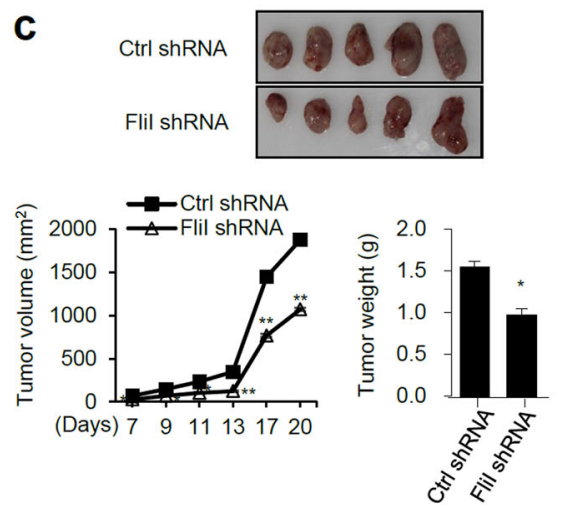

b

$A P C^{\mathrm{min} /+}$

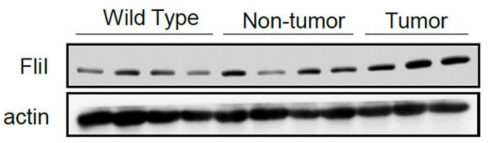

Colitis-associated cancer

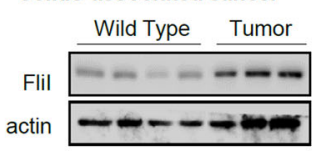

d

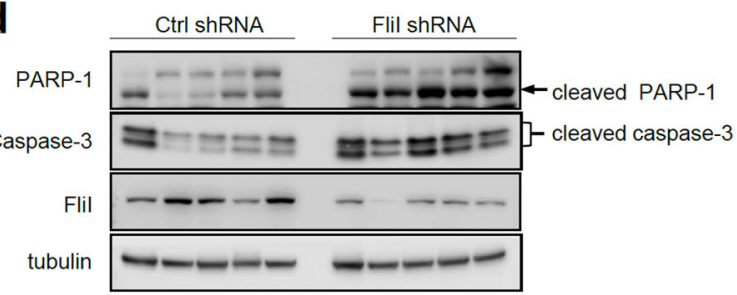

Fig. 1 Flil is overexpressed in colorectal cancer. Flil expression in a colon cancer cell line (a) and colon tissue from APC ${ }^{\text {in/ } /+}$ and colitis-associated cancer mouse models (b) were analyzed by western blotting. c Orthotopic xenografts of Ctrl or Flil-knockdown CT26 cells were detected ( $n=5)$. Images show tumors dissected from the mice. Tumor volume was measured every 2 days, and tumor weight was measured after resection at the end of the experiment. Data are shown as the means \pm SEM. ${ }^{*} p<0.05$ compared with shRNA-Ctrl cells. $\mathbf{d}$ Protein levels of cleaved PARP-1, cleaved caspase-3, and Flil were detected in mouse tumor tissue derived by western blotting.

KD CT26 cells (Fig. 4d). These results strongly suggest that elevation of intracellular $\mathrm{Ca}^{2+}$ decreases the cellular threshold for TG-induced apoptosis in FliI-KD cells.

\section{Knockdown of Flil promotes $\mathrm{ER} \mathrm{Ca}^{2+}$ release through RyR} and alteration of intracellular $\mathrm{Ca}^{2+}$, resulting in cell death

The ER is the primary site of intracellular $\mathrm{Ca}^{2+}$ storage, and $\mathrm{ER} \mathrm{Ca}_{2}{ }^{+}$depletion is the major cause of both $\mathrm{ER}$ stress and ER stress-induced apoptosis ${ }^{35}$. Hence, we postulated that the ER contributes to the increase in intracellular $\mathrm{Ca}^{2+}$ caused by FliI-KD. To test this idea, we determined the concentration of free $\mathrm{Ca}^{2+}$ within the ER by depleting the $\mathrm{ER} \mathrm{Ca}^{2+}$ store with the ionophore ionomycin. As shown in Fig. 5a, ionomycin treatment increased intracellular $\mathrm{Ca}^{2+}$, and the ionomycin-mediated intracellular $\mathrm{Ca}^{2+}$ increase was lower in FliI-KD cells than in control cells. In addition, we measured cytosolic $\mathrm{Ca}^{2+}$ released from the ER by treatment with TG or DBHQ, a specific inhibitor of SERCA, to deplete ER $\mathrm{Ca}^{2+}$. Consistent with the results in Fig. 5a, the intracellular $\mathrm{Ca}^{2+}$ concentration was lower in TG- or DBHQ-treated FliIKD cells (Supplementary Fig. 3a, b). Furthermore, when we exposed FliI-KD cells to a low dose $(1 \mu \mathrm{M})$ of DBHQ for short $(30 \mathrm{~min})$ and long periods $(3 \mathrm{~h}), \mathrm{ER} \mathrm{Ca}^{2+}$ depletion of FliI-KD was observed to occur in a time- dependent manner (Supplementary Fig. 3c), clearly indicating that the elevation in intracellular $\mathrm{Ca}^{2+}$ in FliI-KD cells was caused by the release of $\mathrm{ER} \mathrm{Ca}^{2+}$. Finally, we directly measured the ER $\mathrm{Ca}^{2+}$ concentration using an ER-targeted Cameleon (D1ER) probe. As expected, ER $\mathrm{Ca}^{2+}$ was lower in FliI-KD cells than in control cells (Fig. 5b).

Regulation of intracellular $\mathrm{Ca}^{2+}$ by ER is mainly mediated by $\mathrm{Ca}^{2+}$ reuptake into the ER through SERCA $\mathrm{Ca}^{2+}$ pumps and $\mathrm{Ca}^{2+}$ release through $\mathrm{Ca}^{2+}$ channels, including $\mathrm{IP}_{3} \mathrm{Rs}$ or $\mathrm{RyRs}^{8-10}$. Therefore, we next investigated whether the increase in intracellular $\mathrm{Ca}^{2+}$ in FliI-KD cells was mediated by $\mathrm{ER} \mathrm{Ca}^{2+}$ channels. For these experiments, we used two different $\mathrm{Ca}^{2+}$ channel blockers, dantrolene, tetracaine, and 2-aminoethoxydiphenyl borate (2-APB), which are inhibitors of RyRs and $\mathrm{IP}_{3} \mathrm{Rs}$, respectively. Dantrolene and tetracaine elevated the ionomycininduced increase in intracellular $\mathrm{Ca}^{2+}$ in FliI-KD cells, whereas 2-APB did not (Fig. 5a). Dantrolene and tetracaine also elevated TG-induced and DBHQ-induced intracellular $\mathrm{Ca}^{2+}$ increases in FliI-KD cells (Supplementary Fig. 3a, b). Consistent with the results of intracellular $\mathrm{Ca}^{2+}, \mathrm{ER} \mathrm{Ca}^{2+}$ was also recovered by treatment with dantrolene and tetracaine in FliI-KD cells (Fig. 5b). Furthermore, dantrolene and tetracaine inhibited TG- 


\section{a}

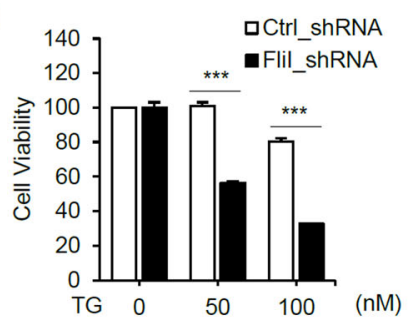

d
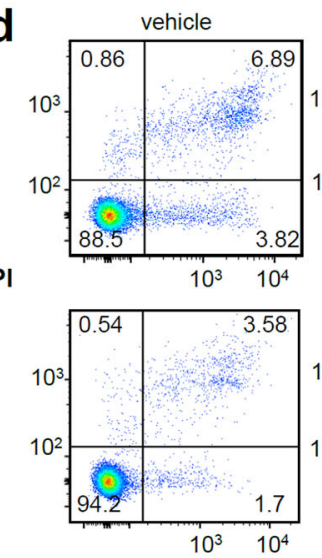
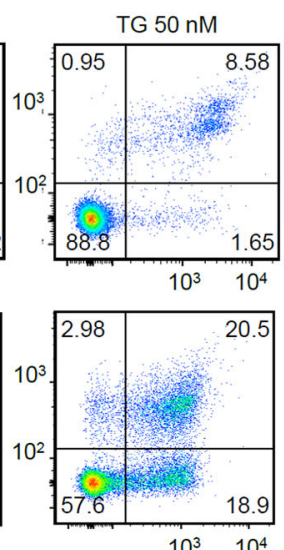

Annexin V
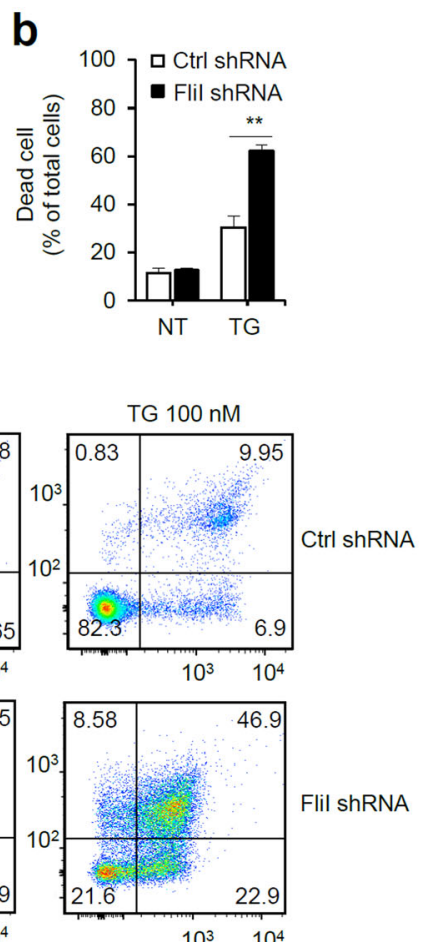

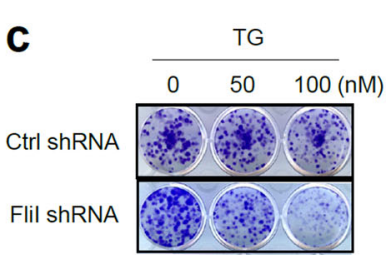

e

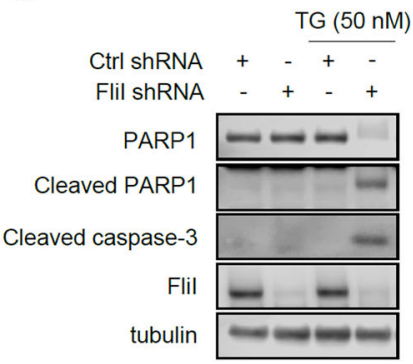

Fig. 2 Knockdown of Flil promotes TG-induced apoptosis in CT26 cells. Cell viability of Flil-KD CT26 cells was measured by MTT assay (a), cell counting assay $(\mathbf{b})$, and colony assay (c) after treatment with TG for $48 \mathrm{~h}$. Data are shown as the mean $\pm \mathrm{SEM} .{ }^{* *} p<0.01$; ${ }^{* *} p<0.001$ shRNA-Ctrl cells vs. shRNA-Flil cells. After Ctrl- and Flil-KD cells were treated with TG for $48 \mathrm{~h}$, they were subjected to annexin V-PI flow cytometry assay (d) and western blotting for PARP-1, cleaved caspase-3, Flil, and tubulin (e). TG thapsigargin.

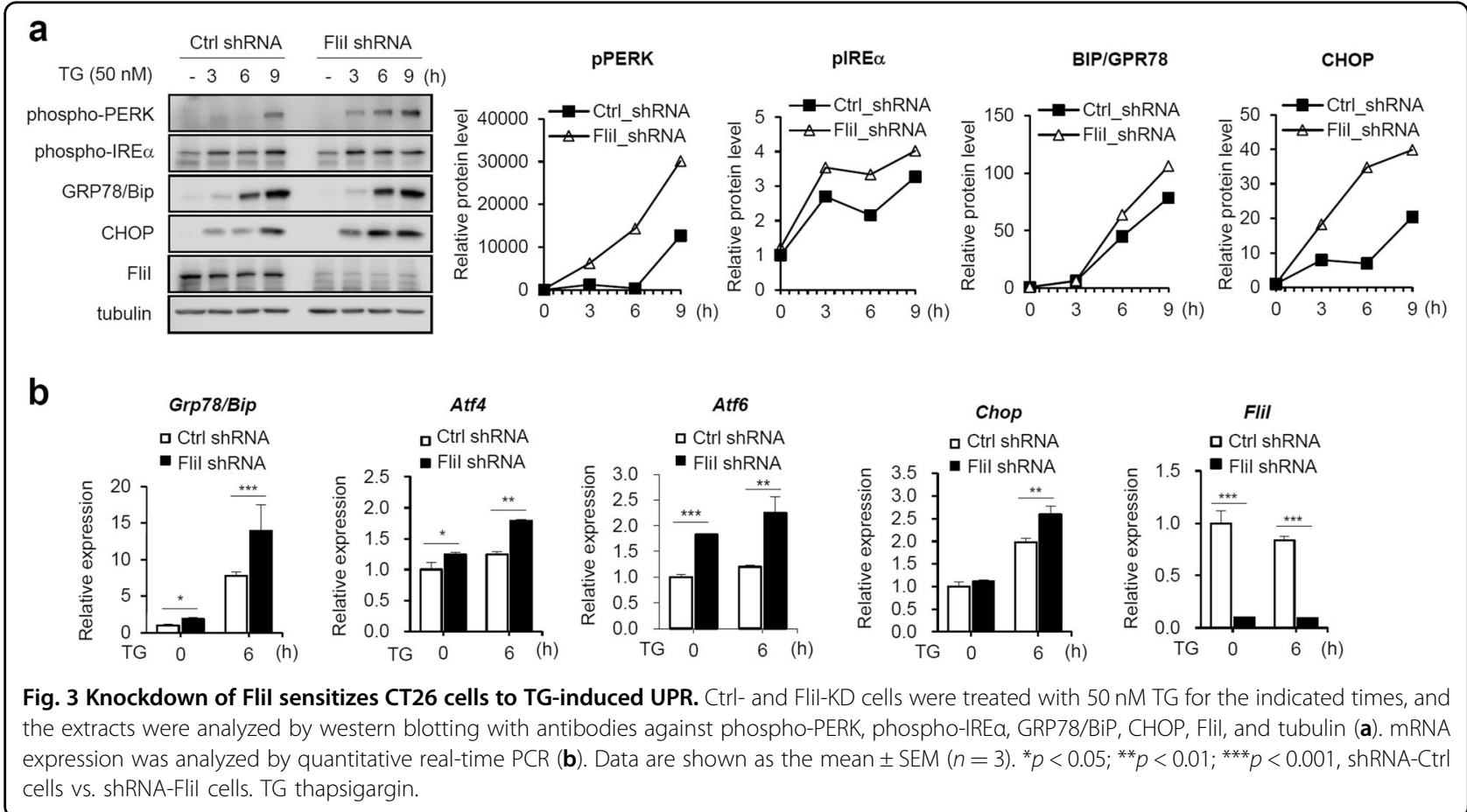



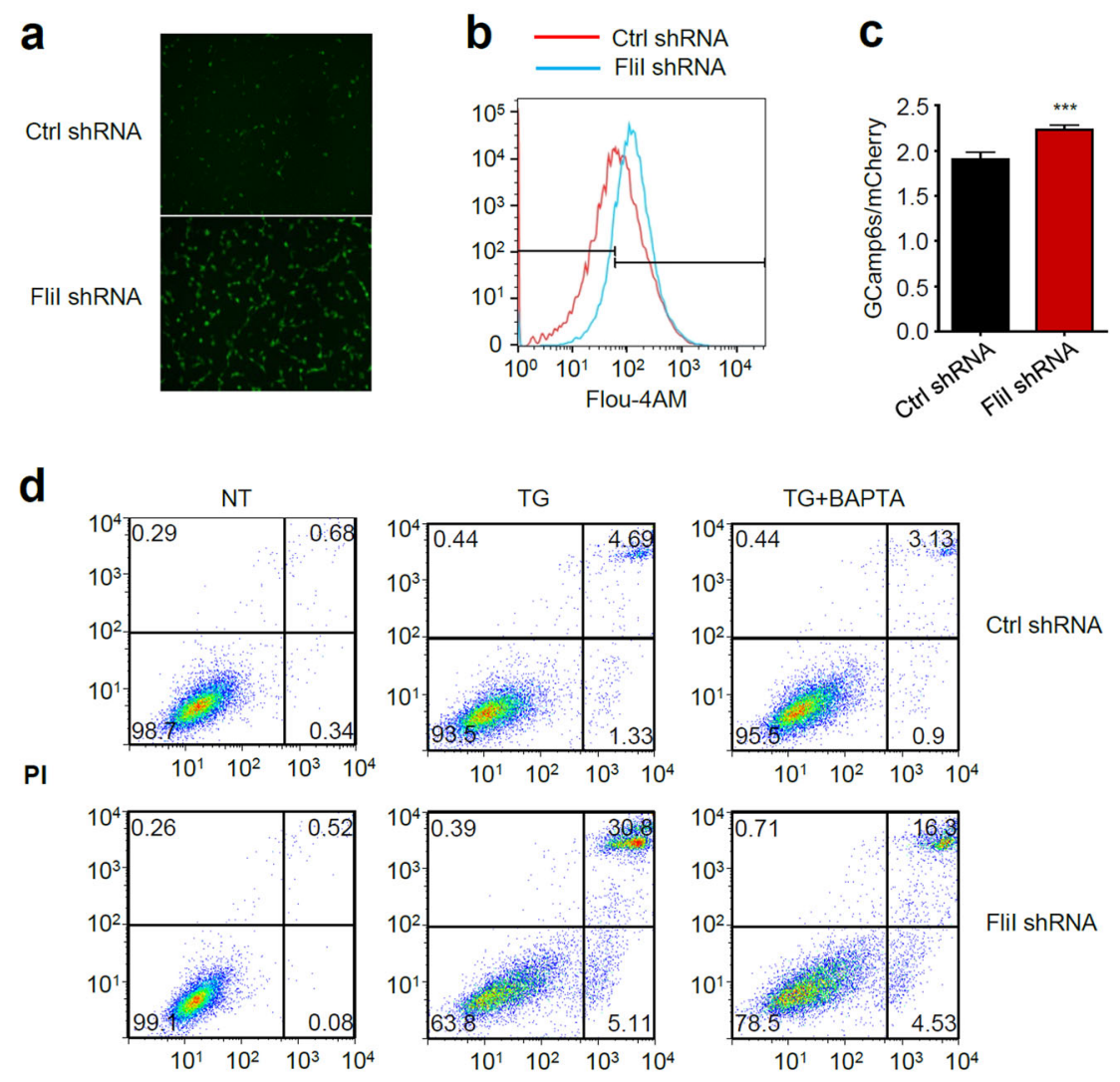

Annexin V

Fig. 4 Knockdown of Flil increases the intracellular $\mathrm{Ca}^{2+}$ concentration in CT26 cells. Ctrl- and Flil-KD cells were incubated with Fluo-4AM for 30 min, and intracellular $\mathrm{Ca}^{2+}$ was (a) imaged on a fluorescence microscope (200x) and (b) measured by FACS analysis. c Ctrl- and Flil-KD cells were transfected with mCherry-GCaMP6s and imaged on a fluorescence microscope. Intracellular $\mathrm{Ca}^{2+}$ levels were monitored based on the ratio of

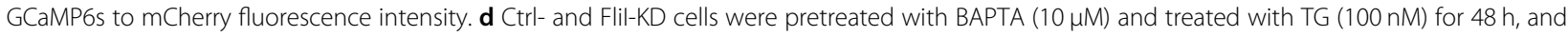
then apoptosis was detected by annexin V-PI flow cytometry assay. TG thapsigargin.

induced expression of ER stress marker proteins (Fig. 5c and Supplementary Fig. 4a). We then tested whether RyRmediated $\mathrm{Ca}^{2+}$ release in FliI-KD cells was associated with TG-induced apoptosis. As shown in Fig. 5d and Supplementary Fig. 4b, inhibition of RyR compromised the increase in TG-induced apoptosis caused by FliI-KD. In addition, the TG-induced increases in the levels of cleaved PARP-1 and caspase-3 in FliI-KD cells were rescued by dantrolene treatment (Fig. 5e). Together, these results strongly suggest that RyR channel-dependent ER $\mathrm{Ca}^{2+}$ release is an essential upstream event in the sensitization of FliI-KD cells to TG-induced apoptosis.

\section{Knockdown of Flil suppresses the expression of sorcin, which protects against ER stress}

To investigate the molecular mechanism by which FliI regulates $\mathrm{Ca}^{2+}$ release through RyRs, we compared the global gene expression profile using RNA sequencing. We focused on sorcin (soluble resistance-related calciumbinding protein) downregulation by FliI-KD (Fig. 6a) because sorcin has been reported to negatively regulate $\mathrm{ER} \mathrm{Ca}^{2+}$ release by mediating RyR and SERCA ${ }^{36,37}$. Then, we hypothesized that downregulated sorcin contributes to the depletion of ER $\mathrm{Ca}^{2+}$ through RyR in FliI-KD cells. To verify this speculation, we overexpressed sorcin in FliI-KD cells and measured ER $\mathrm{Ca}^{2+}$. As shown in Fig. 6b, decreased $\mathrm{ER} \mathrm{Ca}^{2+}$ was recovered by overexpression of sorcin in FliI-KD cells. Furthermore, sorcin overexpression in FliI-KD cells decreased the level of TGinduced ER stress compared with that in vector-expressed FliI-KD cells (Fig. 6c). These results suggest that FliI controls the $\mathrm{ER} \mathrm{Ca}^{2+}$ pool by regulating sorcin expression.

\section{Discussion}

Globally, CRC is the third most commonly diagnosed malignancy and the second leading cause of cancer-related death ${ }^{1}$. In particular, the emergence of refractory disease following chemotherapy continues to be a major contributor to treatment failure and to the high mortality rates observed in advanced CRC. We now know that the 


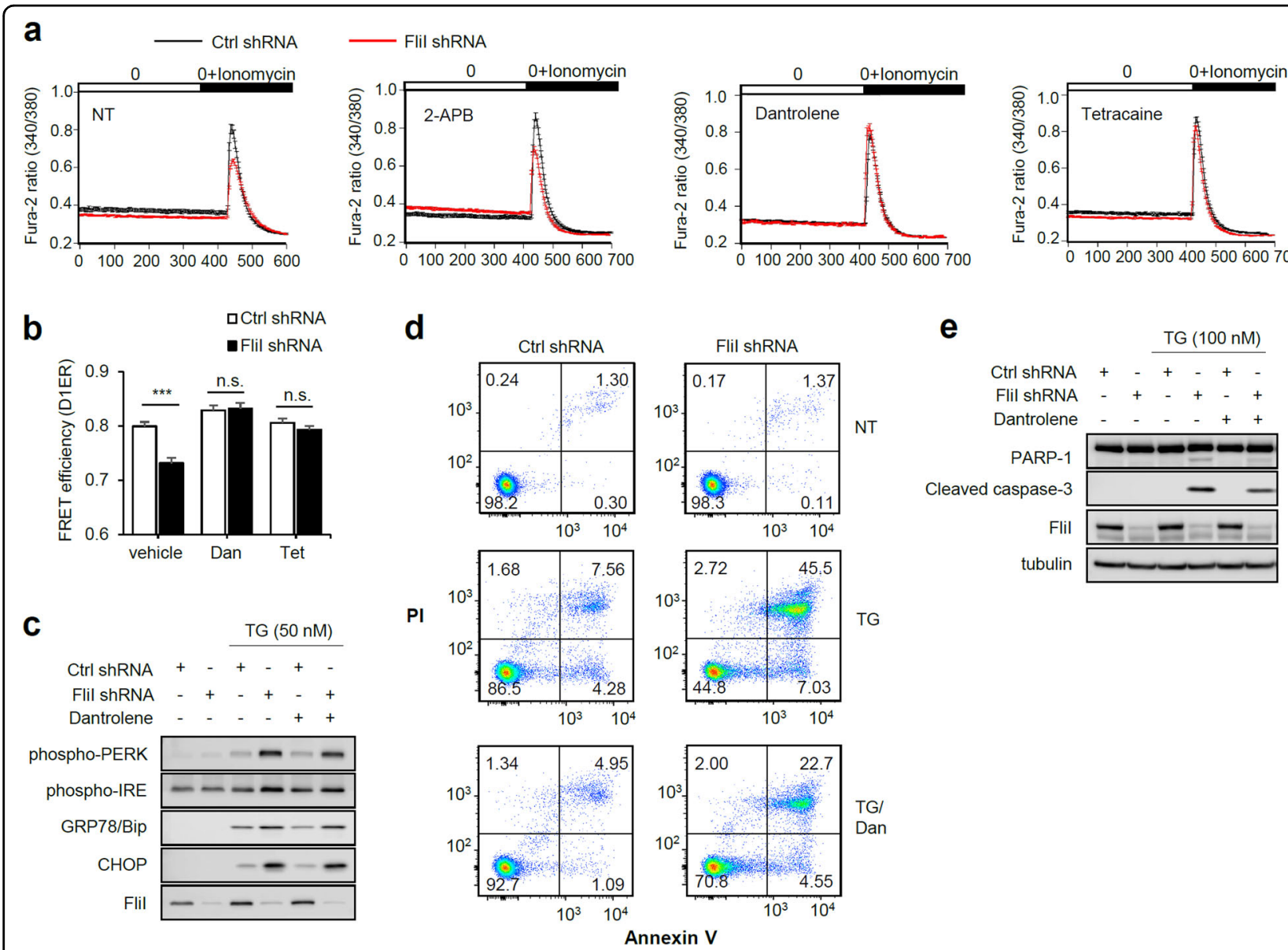

Fig. 5 Knockdown of Flil causes ER Ca ${ }^{2+}$ release through RyRs, which induces ER stress-mediated apoptosis in CT26 cells. a Ctrl- and Flil-KD cells were pretreated for $2 \mathrm{~h}$ with 2-APB $(50 \mu \mathrm{M})$ and dantrolene $(50 \mu \mathrm{M})$ and tetracaine $(50 \mu \mathrm{M})$ and then incubated in Fura-2AM for 30 min. Ratiometric $\mathrm{Ca}^{2+}$ imaging was performed in $0 \mathrm{mM} \mathrm{Ca}{ }^{2+}$ Tyrode's solution with or without $5 \mu \mathrm{M}$ ionomycin, and $\mathrm{Ca}^{2+}$ influx was monitored based on the Fura-2 fluorescence ratio. $\mathbf{b}$ After $24 \mathrm{~h}$ transfection of ctrl- and Flil-KD cells with pcDNA-D1ER, dantrolene $(50 \mu \mathrm{M})$ and tetracaine $(50 \mu \mathrm{M})$ were treated for $2 \mathrm{~h}$, and the FRET signal was measured. Data are shown as the mean \pm SEM. ${ }^{* * *} p<0.001$, shRNA-Ctrl cells vs. shRNA-Flil cells. Dan dantrolene, Tet tetracaine. $\mathbf{c ~ C t r l - a n d ~ F l i l - K D ~ c e l l s ~ w e r e ~ t r e a t e d ~ w i t h ~} 50 \mathrm{nM}$ TG for $6 \mathrm{~h}$ following pretreatment with $50 \mu \mathrm{M}$ dantrolene. Cell lysates were analyzed by western blotting for phospho-PERK, phospho-IREa, GRP78/BiP, CHOP, Flil and tubulin. $\mathbf{d}$, e Ctrl- and Flil-KD cells were pretreated for $2 \mathrm{~h}$ with dantrolene $(20 \mu \mathrm{M})$ and then with TG $(100 \mathrm{nM})$ for $48 \mathrm{~h}$. Apoptosis was detected by annexin V-PI flow cytometry assay (d) and western blotting for PARP-1, cleaved caspase-3, Flil, and tubulin (e). TG thapsigargin, Dan dantrolene.

outcome of anticancer treatment can be influenced by the cancer cell response to cellular stress induced by chemotherapeutic agents. The ER stress response is an adaptive mechanism that protects the cell against environmental stimuli ${ }^{4}$. In addition, the ER has been implicated in promoting apoptosis by releasing calcium, which serves as a secondary messenger for mitochondrion-mediated apopto$\mathrm{sis}^{38}$. In a tumor context, high proliferation rates and accelerated metabolism burden cancer cells with cellular and metabolic stress; therefore, as an adaptation, these cells generally activate the ER stress response to support their own survival $^{39}$. However, this signaling event is a doubleedged sword: although it supports cancer cell survival in an adverse environment, harsh and sustained stress conditions, such as those induced by chemopreventive agents, tip the balance from prosurvival to proapoptosis, culminating in ER stress-mediated cancer cell death ${ }^{39}$. The mechanisms determining the switch from adaptation to cell death signaling under ER stress have not been clearly defined, and the outcome of ER stress signaling may depend on the type, intensity, and duration of the stimulus in relation to active changes in the cellular environment. Despite this lack of clarity, employing strategies to either sensitize or persuade the cancer cells to ER stress may prove to be a useful tool for overcoming drug resistance and proliferation in cancer cells. Notably, van den Brink et al. reported that ER stressinduced differentiation sensitizes colon cancer stem cells to chemotherapy ${ }^{40}$. Many studies have supported the idea that FliI plays important roles in tumor progression by stimulating cell proliferation, inhibiting apoptosis, and promoting 


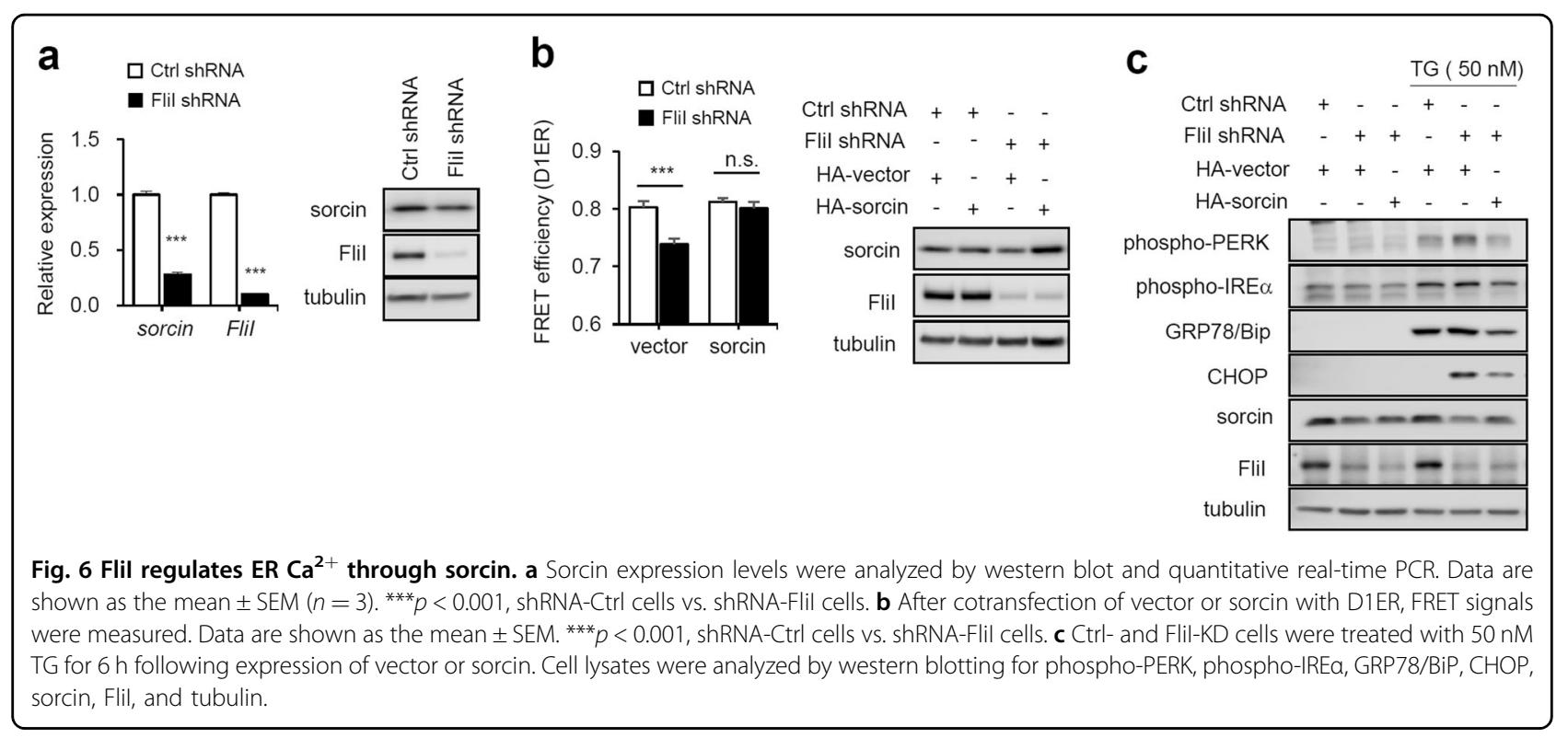

invasion $^{21-23,41}$. In this study, we found that FliI is highly expressed in CRC and that KD of FliI in CT26 cells promoted the induction of ER stress by TG, resulting in apoptosis (Figs. 2, 3). The molecular mechanism by which FliI influences sensitivity to ER stress inducers remains unclear, but we speculate that one important factor is the $\mathrm{Ca}^{2+}$ concentration in the ER and cytosol.

Calcium, stored primarily in the ER, is one of the key regulators of cell survival, but it can also induce apoptosis in response to a variety of pathological conditions ${ }^{29}$. Furthermore, calcium-mediated signaling pathways were implicated in tumorigenesis and tumor progression, including metastasis, invasion, and angiogenesis ${ }^{42,43}$. Conversely, several studies showed that sustained intracellular calcium overload can trigger cell death or deregulate potential calcium-dependent tumorigenic pathways $^{29}$. Recent studies suggest that interfering with the sequestration of calcium into intracellular pools from the ER can trigger apoptosis as part of a stress response $\mathrm{e}^{11,38}$. Thus, the final outcome is determined by the amplitude of the increase and the duration of the change in intracellular calcium level, as well as the nature of the change and the location. In this study, downregulation of FliI in CT26 cells increased intracellular $\mathrm{Ca}^{2+}$ but decreased ER $\mathrm{Ca}^{2+}$ concentration. This $\mathrm{Ca}^{2+}$ depletion was mediated by RyRs (Fig. 5). Thus, depletion of Flil resulted in an increase in the intracellular $\mathrm{Ca}^{2+}$ concentration through $\mathrm{Ca}^{2+}$ release via RyRs, which sensitized cells to ER stress inducers. Thereafter, more rapid and marked increases in intracellular $\mathrm{Ca}^{2+}$ levels triggered apoptosis.

We next asked how FliI regulates $\mathrm{Ca}^{2+}$ release through RyRs. RyRs are modulated directly or indirectly by $\mathrm{Ca}_{\mathrm{V}} 1.1 / 1.2$, various ions, and small molecules and proteins, including $\mathrm{Ca}^{2+}, \mathrm{Mg}^{2+}$, protein kinase A, FK506 binding proteins, calmodulin, $\mathrm{Ca}^{2+} /$ calmodulin-dependent protein kinase II (CaMKII), calsequestrin, and tria$\operatorname{din}^{37}$. Most RyR modulators interact with the cytoplasmic region of the channel. Hence, we first hypothesized that FliI stimulates RyR activation because FliI regulates signaling pathways by interacting with signaling proteins $^{12,13}$. FliI interacts directly with active CaMKII to inhibit its signaling cascade ${ }^{44}$, and CaMKII phosphorylates RyRs in the cytosol, altering their gating properties, leading to $\mathrm{Ca}^{2+}$ release from the $\mathrm{ER}^{45}$. Based on these observations, we postulated that FliI might block the phosphorylation of RyRs by direct interaction with CaMKII, which inhibits RyR activation. However, we did not observe any change in TG-induced UPR signaling or apoptosis when we treated cells with KN-93, a CaMKII inhibitor (data not shown). Therefore, CaMKII activation is not involved in RyR activation by FliI depletion.

Focusing on FliI as a coactivator of transcription factors, we next hypothesized that Flil regulates RyR activation by modulating the expression of genes related to RyRs. Because there was no change in RyR expression in FliI-KD cells (data not shown), we compared global gene expression profiles using RNA sequencing and observed that sorcin (soluble resistance-related calcium-binding protein) was downregulated by depletion of FliI (Fig. 6a). Sorcin is a member of the penta-ERF-hand protein family that localizes to the cytosol, nucleus, plasma membrane, cytoplasmic vesicles, and ER membrane ${ }^{36,44}$. In cardiomyocytes, sorcin interacts with RyRs and SERCA; through these interactions, $\mathrm{Ca}^{2+}$-bound sorcin negatively regulates the release of $\mathrm{Ca}^{2+}$ from the ER and increases the $\mathrm{Ca}^{2+}$ load in the ER by inhibiting RyRs and activating SERCA $^{36,37}$. These changes promote the accumulation of 
$\mathrm{Ca}^{2+}$ in the ER and decrease the level of intracellular $\mathrm{Ca}^{2+}$, thereby preventing ER stress. As expected, overexpression of sorcin in FliI-KD cells increased the ER $\mathrm{Ca}^{2+}$ pool, which recovered TG-induced ER stress (Fig. 6). Sorcin is upregulated under ER stress ${ }^{46}$ and overexpressed in many human tumors, including leukemia, gastric, breast, and ovarian cancers ${ }^{47-50}$, and in chemoresistant cell lines ${ }^{51-55}$. Notably, in this regard, overexpression of sorcin is associated with multidrug resistance (MDR), whereas downregulation of the protein increases sensitivity to antitumor drugs ${ }^{56,57}$. Moreover, sorcin overexpression is correlated with upregulation of MDR1/Pglycoprotein $^{54,57}$, and the P-glycoprotein-dependent MDR phenotype seems to be related to intracellular $\mathrm{Ca}^{2+}$ homeostasis $^{58}$. In fact, we also observed downregulation of MDR1/P-glycoprotein and ABCB1 amplicons, including Dbf4, Crot, and Slc5a40, whose overexpression confers MDR (Supplementary Fig. 5) ${ }^{59}$. Thus, it is intriguing to speculate that sorcin is involved in FliI-mediated regulation of $\mathrm{Ca}^{2+}$ homeostasis, possibly by activating RyRs, which may be responsible for sensitizing cells to ER stress inducer-mediated apoptosis. Further studies are needed to show how FliI regulates sorcin expression.

In conclusion, we have uncovered a novel molecular mechanism in which depletion of FliI increases intracellular $\mathrm{Ca}^{2+}$ concentration in CRC cells by promoting ER $\mathrm{Ca}^{2+}$ release through RyRs, which sensitizes the cells to ER stress inducers and promotes ER stress. Ultimately, this results in ER stress-induced apoptosis. This study provides the first evidence that FliI plays a role in ER stress by controlling $\mathrm{Ca}^{2+}$ homeostasis in CRC cells. In addition, FliI expression is correlated with the expression of sorcin as well as $A B C B 1$ and its amplicons, which are markers of MDR in CT26 cells. Therefore, targeting FliI represents an innovative and effective strategy for the development of cancer therapeutics.

\section{Acknowledgements}

We thank Dr. Yang, Yong Ryoul for kindly providing colorectal cancer tissue (APC ${ }^{\text {mint/- }}$ - and colitis-induced cancer model). This work was supported by a National Research Foundation of Korea (NRF) grant funded by the Korean government (2017R1A2B2007378, 2018R1A5A1024340, 2016H1A2A1909031, 2018R1A5A1024340, 2019R1A2C2002235) and the Ministry of Education (2016R1A6A3A11932338) and by the Korea Health Technology R\&D Project through the Korea Health Industry Development Institute (KHIDI), funded by the Ministry of Health \& Welfare, Republic of Korea (HI18C0412).

\section{Author details}

'Department of Biological Sciences, Ulsan National Institute of Science and Technology (UNIST), Ulsan 689-798, Korea. ${ }^{2}$ Department of Biological Sciences, Center for Genomic Integrity (CGI), Institute for Basic Science (IBS), Ulsan National Institute of Science and Technology (UNIST), Ulsan 689-798, Korea. ${ }^{3}$ Department of Gastroenterology, University of Ulsan College of Medicine, Asan Medical Center, Seoul 05505, Korea

\section{Confict of interest}

The authors declare that they have no conflict of interest.

\section{Publisher's note}

Springer Nature remains neutral with regard to jurisdictional claims in published maps and institutional affiliations.

Supplementary information accompanies this paper at https://doi.org/ 10.1038/s12276-020-0448-3.

Received: 26 December 2019 Revised: 27 April 2020 Accepted: 28 April 2020.

Published online: 5 June 2020

\section{References}

1. Kenum, N. \& Giovannucci, E. Global burden of colorectal cancer: emerging trends, risk factors and prevention strategies. Nat. Rev. Gastroenterol. Hepatol. 16, 713-732 (2019)

2. Wong, R. S. Apoptosis in cancer: from pathogenesis to treatment. J. Exp. Clin. Cancer Res. 30, 87 (2011).

3. Martinon, F. Targeting endoplasmic reticulum signaling pathways in cancer. Acta Oncol. 51, 822-830 (2012).

4. He, B. Viruses, endoplasmic reticulum stress, and interferon responses. Cell. Death. Differ. 13, 393-403 (2006).

5. Schroder, M. \& Kaufman, R. J. The mammalian unfoldedprotein response. Annu. Rev. Biochem. 74, 739-789 (2005).

6. Ariyasu, D., Yoshida, H. \& Hasegawa, Y. Endoplasmic reticulum (ER) stress and endocrine disorders. Int J. Mol. Sci. 18, E382 (2017).

7. Bagur, R. \& Hajnóczky, G. Intracellular $\mathrm{Ca}_{2}^{+}$sensing: its role in calcium homeostasis and signaling. Mol. Cell. 66, 780-788 (2017).

8. Supattapone, S., Worley, P. F., Baraban, J. M. \& Snyder, S. H. Solubilization, purification, and characterization of an inositol trisphosphate receptor. J. Biol. Chem. 263, 1530-1534 (1988).

9. Ellisman, M. H. et al. Identification and localization of nyanodine binding proteins in the avian central nervous system. Neuron 5, 135-146 (1990).

10. Gunteski-Hamblin, A. M., Greeb, J. \& Shull, G. E. A novel Ca2 ${ }^{+}$pump expressed in brain, kidney, and stomach is encoded by an alternative transcript of the slow-twitch muscle sarcoplasmic reticulum Ca-ATPase gene. Identification of cDNAs encoding $\mathrm{Ca}^{+}$and other cation-transporting ATPases using an oligonucleotide probe derived from the ATP-binding site. J. Biol. Chem. 263, 15032-15040 (1988)

11. Wang, H. G. et al. $\mathrm{Ca}_{2}{ }^{+}$-induced apoptosis through calcineurin dephosphorylation of BAD. Science 284, 339-343 (1999).

12. Claudianos, C. \& Campbell, H. D. The novel flightless-l gene brings together two gene families, actin-binding proteins related to gelsolin and leucine-richrepeat proteins involved in ras signal transduction. Mol. Biol. Evo. 12, 405-414 (1995).

13. Marei, $\mathrm{H}$. et al. Differential Rac1 signaling by guanine nucleotide exchange factors implicates FLII in regulating Rac1-driven cell migration. Nat. Commun. 18, 10664 (2016).

14. Kopecki, Z., Cowin, A. J. \& Flightless, I. An actin-remodeling protein and an important negative regulator of wound repair. Int. J. Biochem. Cell. Biol. 40, 1415-1419 (2008).

15. Cowin, A. J. et al. Flightless I deficiency enhances wound repair by increasing cell migration and proliferation. J. Pathol. 211, 572-581 (2007).

16. Jin, J. et al. LRRFIP2 negatively regulates NLRP3 inflammasome activation in macrophages by promoting Flightless---mediated caspase-1 inhibition. Nat. Commun. 4, 2075 (2013).

17. Li, J., Yin, H. L. \& Yuan, J. Flightless-1 regulates proinflammatory caspases by selectively modulating intracellular localization and caspase activity. J. Cell. Biol. 181, 321-333 (2008).

18. Gremm, D. \& Wegner, A. Gelsolin as a calcium-regulated actin filamentcapping protein. Eur. J. Biochem. 267, 4339-4345 (2000).

19. Goshima, M. et al. Characterization of a novel Ras-binding protein Ce-FLI1 comprising leucine-rich repeats and gelsolin-like domains. Biochem. Biophys. Res. Commun. 257, 111-116 (1999).

20. Arora, P. D., Di Gregorio, M., He, P. \& McCulloch, C. A. TRPV4 mediates the $\mathrm{Ca}^{+}$ influx required for the interaction between flightless-1 and non-muscle myosin, and collagen remodeling. J. Cell. Sci. 130, 2196-2208 (2017).

21. Wang, T. et al. Flightless I homolog represses prostate cancer progression through targeting androgen receptor signaling. Clin. Cancer Res. 22, 1531-1544 (2016) 
22. Wu, L. et al. Flightless I homolog negatively regulates ChREBP activity in cancer cells. Int. J. Biochem. Cell. Biol. 45, 2688-2697 (2013).

23. $\mathrm{Xu}, \mathrm{J}$. et al. Identification of Flightless-I as a substrate of the cytokineindependent survival kinase CISK. J. Biol. Chem. 284, 14377-14385 (2009).

24. He, J. P. et al. Flightless-I Blocks p62-Mediated Recognition of LC3 to impede selective autophagy and promote breast cancer progression. Cancer Res. 78, 4853-4864 (2018).

25. Palmer, A. E., Jin, C., Reed, J. C. \& Tsien, R. Y. Bcl-2-mediated alteration in endoplasmic reticulum $\mathrm{Ca}^{2+}$ analyzed with an improved genetically encoded fluorescent sensor. Proc. Natl. Acad. Sci. USA 101, 17404-17409 (2004).

26. Van Rheenen, J., Langeslag, M. \& Jalink, K. Correcting confocal acquisition to optimize imaging of fluorescence resonance energy transfer by sensitized emission. Biophys. J. 86, 2517-2529 (2004).

27. Yang, Y. R. et al. OGA heterozyosity suppresses intestinal tumorigenesis in APC (min+/-) mice. Oncogenesis 3, e109 (2014).

28. Yang, Y. R. et al. Elevated O-GlycNAcylation promotes colonic inflammation and tumorigenesis by modulating NF-kB signaling. Oncotarget 6, 12529-12542 (2015).

29. Brown, J. M. \& Giaccia, A. J. The unique physiology of solid tumors: opportunities (and problems) for cancer therapy. Cancer Res. 58, 1408-1416 (1998).

30. Giorgi, C., Romagnoli, A., Pinton, P. \& Rizzuto, R. Ca2 ${ }^{+}$signaling, mitochondria and cell death. Curr. Mol. Med. 8, 119-130 (2008).

31. Bahar, E., Kim, H. \& Yoon, H. ER stress-mediated signaling: action potential and $\mathrm{Ca}^{+}$as key players. Int. J. Mol. Sci. 17, E1558 (2016).

32. Pinton, P., Giorgi, C., Siviero, R., Zecchini, E. \& Rizzuto, R. Calcium and apoptosis: ER-mitochondria $\mathrm{Ca}^{+}{ }^{+}$transfer in the control of apoptosis. Oncogene $\mathbf{2 7}$ 6407-6418 (2008).

33. Kruman, I., Guo, Q. \& Mattson, M. P. Calcium and reactive oxygen species mediate staurosporine-induced mitochondrial dysfunction and apoptosis in PC12 cells. J. Neurosci. Res. 51, 293-308 (1998).

34. Carafoli, E. Calcium signaling: a tale for all seasons. Proc. Natl. Acad. Sci. USA 99 1115-1122 (2002).

35. Berridge, M. J. The endoplasmic reticulum: a multifunctional signaling organelle. Cell. Calcium 32, 235-249 (2002).

36. Meyers, M. B. et al. Association of sorcin with the cardiac ryanodine receptor. J. Biol. Chem. 270, 26411-26418 (1995).

37. Farrell, E. F., Antaramian, A., Rueda, A., Gomez, A. M. \& Valdivia, H. H. Sorcin inhibits calcium release and modulates excitation-contraction coupling in the heart. J. Biol. Chem. 278, 34660-34666 (2003).

38. Sano, R. \& Reed, J. C. ER stress-induced cell death mechanisms. Biochim. Biophys. Acta 1833, 3460-3470 (2013).

39. Wang, M. \& Kaufman, R. J. The impact of the endoplasmic reticulum proteinfolding environment on cancer development. Nat. Rev. Cancer 14, 581-597 (2014).

40. Wielenga, M. C. B. et al. ER-stress-induced differentiation sensitizes colon cancer stem cells to chemotherapy. Cell. Rep. 13, 489-494 (2015).

41. Kopecki, Z. et al. Cytoskeletal protein Flightless I inhibits apoptosis, enhances tumor cell invasion and promotes cutaneous squamous cell carcinoma progression. Oncotarget 6, 36426-36440 (2015).
42. Lee, J. M., Dabis, F. M., Roberts-Thomson, S. J. \& Monteith, G. R. Remodeling of $\mathrm{Ca}^{+}$signaling in tumorigenesis: role of $\mathrm{Ca}_{2}^{+}$transporter. Am. J. Physiol. Cell. Phyiol. 301, C969-C976 (2011).

43. Lanner, J. T., Georgiou, D. K., Joshi, A. D. \& Hamilton, S. L. Ryanodine receptors: structure, expression, molecular details, and function in calcium release. Cold. Spring Harb. Perspect. Biol. 2, a003996 (2010).

44. Seward, M. E., Easley, C. A. 4th, McLeod, J. J., Myers, A. L. \& Tombes, R. M. Flightless-I, a gelsolin family member and transcriptional regulator, preferentially binds directly to activated cytosolic CaMK-II. Febs. Lett. 582, 2489-2495 (2008).

45. Ai, X., Curran, J. W., Shannon, T. R., Bers, D. M. \& Pogwizd, S. M. Ca2 ${ }^{+} /$Calmodulin-dependent protein kinase modulates cardiac ryanodine receptor phosphorylation and sarcoplasmic reticulum $\mathrm{Ca}^{+}$leak in heart failure. Circ. Res. 97, 1314-1322 (2005).

46. Landriscina, M. et al. Mitochondrial chaperone Trap1 and the calcium binding protein Sorcin interact and protect cells against apoptosis induced by antiblastic agents. Cancer Res. 70, 6577-6586 (2010).

47. Pomeroy, S. L. et al. Prediction of central nervous system embryonal tumour outcome based on gene expression. Nature 415, 436-442 (2002).

48. Tan, $Y$. et al. Expression of sorcin predicts poor outcome in acute myeloid leukemia. Leuk. Res. 27, 125-131 (2003).

49. Yokota, T. et al. Identification of histological markers for malignant glioma by genome-wide expression analysis: dynein, alpha-PIX and sorcin. Acta Neuropathol. 111, 29-38 (2006).

50. Nagpal, J. K. \& Das, B. R. Identification of differentially expressed genes in tobacco chewing-mediated oral cancer by differential display-polymerase chain reaction. Eur. J. Clin. Invest. 37, 658-664 (2007).

51. Meyers, M. B., Schneider, K. A., Spengler, B. A., Chang, T. D. \& Biedler, J. L. Sorcin (V19), a soluble acidic calcium-binding protein overproduced in multidrugresistant cells. Identification of the protein by anti-sorcin antibody. Biochem. Pharmacol. 36, 2373-2380 (1987).

52. Van der Bliek, A. M. et al. Genes amplified and overexpressed in human multidrug-resistant cell lines. Cancer Res. 48, 5927-5932 (1988).

53. Padar, S. et al. Differential regulation of calcium homeostasis in adenocarcinoma cell line A549 and its Taxol-resistant subclone. Br. J. Pharmacol. 142 305-316 (2004).

54. Zhou, Y. et al. Sorcin, an important gene associated with multidrug-resistance in human leukemia cells. Leuk. Res. 30, 469-476 (2006).

55. Yang, Y. X., Chen, Z. C., Zhang, G. Y., Yi, H. \& Xiao, Z. Q. A subcelluar proteomic investigation into vincristine-resistant gastric cancer cell line. J. Cell. Biochem. 104, 1010-1021 (2008).

56. Kawakami, M. et al. Knock-down of sorcin induces up-regulation of MDR1 in HeLa cells. Biol. Pharm. Bull. 30, 1065-1073 (2007).

57. He, Q. et al. Overexpression of sorcin results in multidrug resistance in gastric cancer cells with up-regulation of P-gp. Oncol. Rep. 25, 237-243 (2011).

58. Sulová, Z. et al. Does any relationship exist between P-glycoprotein-mediated multidrug resistance and intracellular calcium homeostasis. Gen. Physiol. Biophys. 28, F89-F95 (2009).

59. Genovese, I., llari, A., Assaraf, Y. G., Fazi, F. \& Colotti, G. Not only P-glycoprotein: amplification of the $A B C B 1$-containing chromosome region 7q21 confers multidrug resistance upon cancer cells by coordinated overexpression of an assortment of resistance-related proteins. Drug. Resist. Updat. M32, 23-46 (2017). 